\title{
MANDARIN STOPS CLASSIFICATION BASED ON RANDOM FOREST APPROACH
}

\author{
Chi-Yueh Lin, Hsiao-Chuan Wang \\ Department of Electrical Engineering, National Tsing Hua University, Hsinchu \\ bancolin@gmail.com, hcwang@ee.nthu.edu.tw
}

\begin{abstract}
The non-stationary behavior makes stops classification one of worthy examining subject in the speech community. Over several decades, many researchers have sorted out a list of acoustic properties that are useful to identify a stop. In this paper, we extract features that are sufficient to represent the important acoustic properties of stops, like statistic moments of the burst spectrum. In combining a recent developed learning approach, the random forest, we conduct a 6-way classification task to classify Mandarin stops. After a series of bootstrap trials, experimental results demonstrate the superior performance of random forest on the stop classification task over some well-known approaches.
\end{abstract}

Index Terms - stop classification, random forest

\section{INTRODUCTION}

The research on consonants has always been an attractive topic in the speech community. Stops, the most special category among consonants, leave many challenging problems awaiting enthusiastic researchers to solve. The nonstationary, transient nature of stops makes many analytic approaches hard to apply directly. Furthermore, stops in different languages reveal different acoustic properties. Take 6 stops in Taiwan Mandarin $\left(/ \mathrm{p} /, / \mathrm{t} /, / \mathrm{k} /, / \mathrm{p}^{\mathrm{h}} /, / \mathrm{t}^{\mathrm{h}} /, / \mathrm{k}^{\mathrm{h}} /\right)$ for example, all of these stops voiceless. The compartment is based on whether or not the stop has aspiration or not. This property is different from stops in English, for which also have voiced/voiceless distinction. That observation implies that acoustic properties of stops observed in one language may not be applied directly to another language.

After several decades of research and study, phonetic cues contribute to a stop classification task can be mainly obtained from two segments of a stop: the burst release and the onset of voicing [1][2][3][4][5]. From the view of identifying place of articulation, useful cues from burst release are burst amplitude, spectrum shape, and peak location of the spectrum. From the onset of voicing, cues are mainly from the trends that formants transiting. In addition, the duration between the stop burst and the voicing onset is the most important cue to discriminate aspirated stops from unaspirated ones. Many perceptual researches reveal the decision boundary lies around $2025 \mathrm{~ms}$. Some researchers even assert this boundary is innate.
The results of stop classification reported in the literatures vary from $50 \%$ up to almost perfection [1][2][3][6]. The numbers are greatly influenced by recording conditions. If the stops are pronounced formally in a quiet, high SNR environment, high classification rate can be achieved. However, if the recording is interfered with some background noise, some major cues used to classify stops will become unreliable [7][8]. Even in a natural or spontaneous speech, the stop burst may sometimes be weakly presented, or even worse, the burst is almost omitted.

In this paper, we discuss some major subjects in the task of stop classification, like what kind of features to be used and which type of classifier to be applied. Our experimental results demonstrate the usefulness of LPC-spectrum derived features to the stop classification task. And a comparison between different kinds of classifiers reveals the superiority of random forest over some well-known approaches. In the following sections, brief explanations of the extracted features are given first. Then it comes the introduction to the random forest [10] and other learning approaches. In the experiment section, we will explain how speech materials are prepared and how experiments are conducted. Finally, experimental results and some discussion conclude this paper.

\section{STOP RELATIVE FEATURES}

Most features used in classifying stops are derived from spectrum, especially from the short segment of stop burst. But not all of features mentioned in the literature are useful to the Mandarin stops because six stops in Mandarin are voiceless. Here we use spectral features like center of gravity, variance, skewness, and kurtosis of the burst spectrum. Furthermore, we also take the slope and the peak location of the burst spectrum into consideration. All spectral features used in this paper are computed from LPC spectrums. In the following subsections, introduction to these spectral features is visited.

\subsection{Statistic Moments of Spectrum}

Firstly, the computed LPC spectrum is normalized as a probability distribution. Denote $p(1), \cdots, p(K)$ the spectral magnitudes at discrete frequencies $f_{1}, \cdots, f_{K}$. Each magnitude $p(k)$ is normalized as eqn.(1) shown so that $\sum \bar{p}(k)=1$. Then the center of gravity of the burst spectrum, $L_{c o g}$, is computed based on this normalized spectrum. After $L_{c o g}$ is 
obtained, three statistic moments relative to $L_{c o g}$ are progressively derived. They are variance, skewness, and kurtosis of the burst spectrum.

$$
\bar{p}(k)=\frac{p(k)}{\sum_{j=1}^{K} p(j)} \quad, \text { where } k=1, \cdots, K
$$

The center of gravity of the spectrum is defined as

$$
L_{\text {cog }}=f_{1} \cdot \bar{p}(1)+\cdots+f_{K} \cdot \bar{p}(K)
$$

. Once $L_{c o g}$ was calculated, other statistic moments can be calculated as follows. The formula of the variance of the spectrum is

$$
L_{v a r}=\left(f_{1}-L_{\operatorname{cog}}\right)^{2} \bar{p}(1)+\cdots+\left(f_{K}-L_{c o g}\right)^{2} \bar{p}(K)
$$

The third moment, skewness, is calculated using

$$
\begin{aligned}
\tilde{L}_{\text {skew }} & =\left(f_{1}-L_{\text {cog }}\right)^{3} \bar{p}(1)+\cdots+\left(f_{K}-L_{\text {cog }}\right)^{3} \bar{p}(K) \\
L_{\text {skew }} & =\tilde{L}_{\text {skew }} /\left(L_{\text {var }}\right)^{1.5}
\end{aligned}
$$

And the fourth moment, kurtosis, is obtained by

$$
\begin{aligned}
\tilde{L}_{\text {kurt }} & =\left(f_{1}-L_{\text {cog }}\right)^{4} \bar{p}(1)+\cdots+\left(f_{K}-L_{\text {cog }}\right)^{4} \bar{p}(K) \\
L_{\text {kurt }} & =\left[\tilde{L}_{\text {kurt }} /\left(L_{\text {var }}\right)^{2}\right]-3
\end{aligned}
$$

The above $L_{\text {skew }}$ and $L_{\text {kurt }}$ are called dimensionless versions of original skewness and kurtosis. The purpose of dimensionless versions is try to normalize the third and fourth moments with respect to the shift in center frequency and frequency scale that can occur between different speakers.[2]

\subsection{The slope and the peak of the spectrum}

Based on the discussion of stop acoustic properties [1], place of articulation can be classified according to the gross shape of spectrum. Labial stops reveal a falling trend, which means most power distributes over lower frequency bands. On the contrary alveolar stops have more power in higher frequency bands, thus demonstrate a rising trend. Different from the previous two categories, velar stops have a "compact" spectrum. Compact means the spectrum peak roughly lies in the mid frequency bands and displays falling trends toward either ends. The location of spectrum peak is another useful cue to discriminate between different stops. The spectrum peak is located through a simple computation.

$$
L_{\text {peak }}=\underset{k}{\operatorname{argmax}}\{p(k) \mid k=1, \cdots, K\}
$$

\subsection{Log-energy}

Normalized log-energy of each frame is calculated as follows. Given a sequence of frames, calculate log-energy of each frame. Then set the maximum of these log-energies to 1 , then log-energies of other frames are adjusted their level according to this new maximum value. The operation can be summarized below: [9]

$$
E^{\prime}(n)=E(n)-\max \{E(n) \mid n=1, \cdots, N\}+1
$$

, where $E(n)$ is the original log-energy of frame $n$ and $E^{\prime}(n)$ is its adjusted version. Besides, the conventional delta and delta-delta parameters of normalized log-energies are also calculated.

\subsection{Voice/Vowel Onset Time (VOT)}

The duration between the stop burst and the vocalic onset is marked as VOT usually. According to Edwards' paper [3], there exist two terms relating to VOT and we think it is important to clarify here. The first one is Voice Onset Time, the onset time is marked according to the appearance of first glottal vibration. The second definition, Vowel Onset Time, is primarily based on the appearance of higher formants of the following Mandarin final. The measured duration of vowel onset time is usually slightly longer than that of voice onset time. In this paper we follow the vowel onset time definition.

\section{CLASSIFICATION MACHINES}

\subsection{Random Forest}

Random forest was originally proposed by Breiman in the early 2000 [10], as a combination of two conventional approaches: classification and regression tree (CART) and bootstrap and aggregation (bagging). In a broaden view, a random forest consists of many decision trees, for the number may be up to hundreds or thousands. The operation of a random forest is almost identical to a simple decision tree. Here we take classification task for example. The testing case is fed down through each decision tree in the forest, for each tree makes its local decision. Then the final decision is determined through a majority vote. On the other hand, the construction phase of a random forest is far more sophisticated. Suppose there are $N$ cases in the training set and each case is composed of $M$ variates. In growing each tree, we randomly pick $N$ cases with replacement from training set. By this strategy, roughly $63 \%$ of the picked cases are distinct $\left(1-(1-1 / N)^{N} \approx 63 \%\right.$ as $N$ increases). The node splitting criterion, however, is only based on $m$ randomly chosen variates, where $m \ll M$. That means we divide the training cases into two subgroups by the variate which reduces Gini diversity most among those $m$ randomly chosen variates. In the following node splittings, we again randomly choose another set of $m$ variates. The procedure is repeated until the tree is grown. Notice each tree in a random forest is grown without any pruning, which is the common technique in a CART.

The remaining $37 \%$ cases, which are specifically termed as out-of-bag (OOB) cases, play the role like a testing set. Utilization of OOB cases is another feature of random forest. Its main purpose is to estimate the unbiased error rate of the decision. The case which is not used to grow the tree is 
served as a testing case of that tree. Broadly speaking, each case in the training set $\mathrm{S}$ is the testing case of $37 \%$ of trees in the forest. While estimating error rate using OOB cases, put each OOB case $x_{n}$ down to those trees that do not use $x_{n}$ as training case. The final decision is still based on a majority vote, but only from the results made by those $37 \%$ of trees. This error rate estimation scheme is another advantage of the random forest. It explicitly points out no testing set should be arranged beforehand. Error rate is estimated internally within the random forest.

\subsection{Support Vector Machine}

Support vector machine has its good reputation for its classification ability over some conventional approaches. Different from the widely used Gaussian mixture model (GMM) in the speech community, for which is categorized under the generative models. SVM, on the contrary, stands on the opposite side of generative models, termed discriminative models. Unlike Gaussian mixture model, support vector machine does not try to model the data distribution. Instead it uses kernel trick to find data examples lying around the class boundaries that can help to specify the decision boundaries. SVM also utilizes the large margin concept which often demonstrates a better performance to a classification task.

\subsection{Classification and Regression Tree}

Classification and regression tree (CART) was also proposed by Breiman about 20 years ago. Its simplicity, fast training, and interpretable outcome makes it one of popular approaches since then. As mentioned earlier, the difference between CART and RF can be summarized as follows. (1) CART has only one single tree whereas RF consists of hundred or thousand trees. (2) The variate that CART uses to split the node is chosen by comparing all variates. RF chooses the variate only among those randomly selected variates. (3) CART usually needs pruning to avoid the over-fitting problem; however, no pruning is needed in RF because RF does not suffer from the over-fitting problem.

\subsection{Gaussian Mixture Model}

Gaussian mixture models can be seen almost everywhere in the speech processing community. The unknown data distribution is approximated by a set of Gaussian distributions and each Gaussian distribution only has three set of parameters to be estimated. They are weights, mean vector, and covariance matrix of the mixture component. Common initialization scheme used in GMM can be random initialization, k-means, or central splitting.

\section{EXPERIMENTS AND DISCUSSION}

Stop segments used in the experiment are manually extracted from a subset of TCC-300 Mandarin speech database. The whole TCC-300 database consists of about 27,000 utterances
Table 1. Number of Mandarin stops used in the experiment.

\begin{tabular}{lcccccc}
\hline Stop & $/ \mathrm{p} /$ & $/ \mathrm{t} /$ & $/ \mathrm{k} /$ & $/ \mathrm{p}^{\mathrm{h}} /$ & $/ \mathrm{t}^{\mathrm{h}} /$ & $/ \mathrm{k}^{\mathrm{h}} /$ \\
\hline $\begin{array}{l}\text { Number } \\
\text { of cases }\end{array}$ & 265 & 164 & 178 & 96 & 181 & 171 \\
\hline
\end{tabular}

which roughly equates 26 hours. Speech utterances in the TCC-300 database were spoken by 300 speakers in a quasimonologue style in an office-like environment. The sampling rate and quantization bit are $16,000 \mathrm{~Hz}$ and 16-bit, respectively. The SNR of recording is about $23 \mathrm{~dB}$. Only 1,001 stop segments from 9 females and 7 males were extracted for our experiment. The numbers of each Mandarin stops are listed in the Table 1. Each segment, actually in the unit of Mandarin syllable, consists of a desired stop portion and its following Mandarin final. In order to make sure the stop burst to be analyzed, the starting point of each segment is about $10 \mathrm{~ms}$ prior to the stop burst. Furthermore, the position of the burst release and the position of the following final(vowel) onset are annotated manually.

Extracted segments are first filtered by a $1-0.97 z^{-1}$ preemphasis filter, then are analyzed by a $10 \mathrm{~ms}$ Hamming window with a $2 \mathrm{~ms}$ frame rate. 24 linear prediction coefficients are calculated by Levinson-Durbin algorithm for each frame. A LPC spectrum with 512-point resolution is derived by the previously computed LPCs. The features mentioned in the section 2 are obtained from these LPC spectrums from the frequency range between $20 \mathrm{~Hz}$ and $7500 \mathrm{~Hz}$.

After all required features are obtained, we use 10 frames that begin from the stop burst and 5 frames that around the vowel onset to represent the segment information. These 15 frames of features are concatenated in tandem to form a 121 dimensional super-vector. These super-vectors are pooled together to form the training set of RF and CART, whereas SVM and GMM use the z-normalized version. The z-normalization is practiced as following: each dimension is subtracted by its mean and then divided by its standard deviation.

The experiment is run as follows: At each trial, bootstrap 1,001 training samples from the original data set $\mathrm{S}$. The proportion of 6 stops in this bootstrapped training set is kept as equal as possible to that of the original data set. Examples that are not selected in the bootstrapping are grouped together to form the testing set for that trial.100 trials are executed and the benchmark is based on the average performance of these 100 trials. The reason for an arrangement of bootstrapped samples is twofold. The first is to reduce the benchmark variance because the number of samples we used is not much. The second reason is for a comparison between different learning machines because SVM, CART, and GMM, unlike RF, need an external testing set. The error rate estimation of RF using OOB cases, although not shown here, is according with the bootstrapped result, but slightly $2 \%$ better because in this situation the RF can use full data set to build trees.

Table 2 summarized the performance of mentioned clas- 
sifiers. We set RF consist of 1,500 tress and set the number of randomly chosen variates $m$ be 13 . The number of components in a GMM is 4 and covariance matrix in each component is set to be diagonal. The superiorities of RF and SVM over CART and GMM are obvious. The reason that RF over SVM comes from the tree-based approach can put more importance on some most discriminative variates, e.g. VOT. For those non tree-based approaches, there are no explicit mechanisms to give some variates higher precedence over the others. When the feature vector is composed of many different kinds of features that have different degree of discriminations, a tree-based approach has a chance to take advantage of the variate that has more authority. The same effect can also be found from the result of a 2-way unaspirated/aspirated stops classification, as shown in Table 3. The discrimination between unaspirated and aspirated stops reflects directly the influence of whether the VOT had been emphasized or not. In the experiment the number of trees in the RF is varied to find an optimal performance. We find there is no much change in our results when the number varies from 1,200 to 2,000. As for the number of randomly chosen variates in each splitting, $m$, according to Breiman's paper [10], $\sqrt{M}$ is a good starting point to seek through.

Not surprisingly, both RF and CART sift the VOT the most important factor to the 6-way stop classification. If we eliminate the VOT and repeat the experiment, the performance of RF and SVM degrade to around 75\%, GMM drops to $62 \%$, CART however deteriorates sharply to $55 \%$. Exclusion of the VOT brings more harmful impact on tree-based approaches just because their performances rely so heavily on the VOT. On the other hand, for those approaches that implicitly treat every variate equally, e.g. SVM and GMM, have less degree of degradation. Besides VOT, both RF and CART have agreed that the next two ranks of features are the skewness and the peak location of the spectrum at the burst release. This ranking is agreed with [3] except Edwards did not take the skewness into consideration.

We also conduct some experiments to compare the stop classification performance between RF and HMM. With conventional 13-order MFCC and log energy plus their delta and delta-delta as features, the whole segment of stop was modeled by a 3-state left-to-right HMM. Each state has 4 GMMs with diagonal covariance matrix each. The parameters of each HMM are estimated with 20 iterations by Baum-Welch algorithm in a maximum likelihood manner. Because RF has no ability to model a sequence of data like a HMM, we still follow the procedure in the former experiment: Use 10 frames of MFCC begin at the burst;combine another 5 frames of MFCC around the vowel onset, to form a 630-dim supervector for each stop segment. The performances of these two approaches are almost identical, but only have $73 \%$ of classification rates. From above results we find that LPC-spectrum derived features are more suitable than MFCCs for a stop classification task under the condition that segment boundaries are known. However, MFCCs still have their advantage of modeling speech dynamics under the framework of HMMs.
Table 2. Average 6-way classification rates of different learning machines over 100 bootstrapped trials.

\begin{tabular}{lccccccc}
\hline Stop & $/ \mathrm{p} /$ & $/ \mathrm{t} /$ & $/ \mathrm{k} /$ & $/ \mathrm{p}^{\mathrm{h}} /$ & $/ \mathrm{t}^{\mathrm{h}} /$ & $/ \mathrm{k}^{\mathrm{h}}$ & $\begin{array}{l}\text { Avg. } \\
\text { Rate }\end{array}$ \\
\hline $\begin{array}{l}\text { RF } \\
(1500,13)\end{array}$ & $73 \%$ & $87 \%$ & $81 \%$ & $74 \%$ & $91 \%$ & $76 \%$ & $82 \%$ \\
\hline SVM & $75 \%$ & $84 \%$ & $81 \%$ & $69 \%$ & $87 \%$ & $71 \%$ & $80 \%$ \\
\hline CART & $61 \%$ & $72 \%$ & $68 \%$ & $60 \%$ & $72 \%$ & $65 \%$ & $68 \%$ \\
\hline GMM & $62 \%$ & $73 \%$ & $59 \%$ & $66 \%$ & $76 \%$ & $63 \%$ & $57 \%$ \\
\hline
\end{tabular}

Table 3. Average unaspirated/aspirated classification rates of different learning machines over 100 bootstrapped trials.

\begin{tabular}{cccccc}
\hline $\begin{array}{c}\text { Learning } \\
\text { Machines }\end{array}$ & $\begin{array}{c}\text { Unaspi- } \\
\text { rated }\end{array}$ & Aspirated & $\begin{array}{c}\text { Learning } \\
\text { Machines }\end{array}$ & $\begin{array}{c}\text { Unaspi- } \\
\text { rated }\end{array}$ & Aspirated \\
\hline RF & $96 \%$ & $95 \%$ & CART & $95 \%$ & $91 \%$ \\
\hline SVM & $94 \%$ & $90 \%$ & GMM & $83 \%$ & $89 \%$ \\
\hline
\end{tabular}

How to incorporate advantages of these features and learning methods needs a further investigation.

\section{ACKNOWLEDGEMENT}

This research was sponsored by the National Science Council, Taiwan, under contract number NSC96-2221-E-007-035.

\section{REFERENCES}

[1] R.D. Kent, and C. Read, "The Acoustic Analysis of Speech," Singular Publishing Group, Inc., 1992.

[2] K. Forrest, G. Weismer, P. Milenkovic, and R.N. Dougall, "Statistical analysis of word-initial voiceless obstruents: Preliminary data," in J. Acoust. Soc. Am. 84(1), pp. 115-123, July 1988.

[3] T.J. Edwards, "Multiple features analysis of intervocalic English plosives," in J. Acoust. Soc. Am. 69(2), pp. 535-547, Feb. 1981.

[4] M.F. Dorman, "Relative spectral change and formant transitions as cues to labial and alveolar place of articulation," in J. Acoust. Soc. Am. 100(6), pp. 3825-3830, December 1996.

[5] H. Fujisake, and M. Tominaga, "Automatic recognition of voiced stop consonants in CV and VCV utterances," in IEEE ICASSP 1982, pp.1996-1999.

[6] H.C. Wang, L.C. Liu, and L.M. Lee, "A study on the automatic recognition of voiceless unaspirated stops," in J. Acoust. Soc. Am. 89(1), pp. 461-464, January 1991.

[7] P. Niyogi, and M. M. Sondhi, "Detecting stop consonants in continuous speech," in J. Acoust. Soc. Am. 111(2), pp.10631076, February 2002.

[8] G. Parikh, and P.C. Loizou, "The influence of noise on vowel and consonant cues, " in J. Acoust. Soc. Am. 118(6), pp.38743888, December 2005.

[9] S. Young, and et al., "The HTK book for HTK version 3.2.1."

[10] L. Breiman, "Random Forests," in Machine Learning 45(1), pp. 5-32, 2001. 